\title{
Microstructure formation during thermomechanical processing in Ti-17 alloy
}

\author{
Elango Chandiran ${ }^{\mathrm{a}}$, Goro Miyamoto ${ }^{\mathrm{a}}$, Tadashi Furuhara ${ }^{\mathrm{a}}$, \\ a Institute for Materials Research, Tohoku University, Sendai, Japan \\ *furuhara@imr.tohoku.ac.jp
}

\begin{abstract}
$\underline{\text { Abstract }}$
The microstructure evolution during solution treatment at (alpha+beta) two phase region and subsequent aging process at $620{ }^{\circ} \mathrm{C}$, after compressing the specimen in beta structure to different strain $(0-70 \%)$ followed by cooling to room temperature has been investigated. Deformation strain hardly affects the total volume fraction of alpha phase after the solution treatment and aging process. On the other hand, with an increase in deformation strain, the volume fraction of the primary alpha phase increased although secondary alpha phase decreased, during the aging process, scuhdecrease in secondary alpha phase volume fraction results in a decrease in the strength. The loss of coherency of alpha/beta interphase boundaries at higher deformation strain promoted by dynamic recovery increases the interfacial mobility; which results in faster growth of primary alpha, thereby decreases secondary alpha precipitation.
\end{abstract}

\section{$\underline{\text { 1. Introduction }}$}

Ti-17(Ti-5Al-2Sn-2Zr-4Mo-4Cr(mass\%)) is a metastable beta $(\beta)$ Ti-alloy primarily used in the aerospace industry for parts such as compressor and turbine discs, because of its excellent combination of tensile strength, fracture toughness and combined strength, and low cycle fatigue capabilities $[1,2]$. The typical processing route followed for such titanium alloys consists of, series of hot working in $\beta$ structure above or below the $\beta$ transus temperature $\left(\mathrm{T}_{\beta}\right)$ and followed by (alpha+beta) solution treatment and the aging process. During this processing, the precipitation of alpha $(\alpha)$ phase occurs, whose morphology, volume fraction, size and distribution depend upon the processing parameters, which inherently influence the properties of the Ti-17 alloy [3, 4]. So, it is essential to understand the microstructure evolution during thermomechanical processing to control the mechanical properties of Ti-17 alloy. Most of the previous studies were focused on the microstructure evolution during hot compression [5,6,7] and subsequent cooling to room temperature $[8,9]$. However, very few researches have been conducted to understand the effect of hot deformation conditions on the microstructure evolution during subsequent solution treatment and aging. In the present study, the effect of deformation strain on the microstructure evolution during solution treatment and aging is investigated.

\section{Materials and experiments}

The chemical composition of the Ti-17 alloy used in this study is Ti-5.1Al-3.9Mo-4.0Cr-2.0Zr-2.0Sn-0.1O (in mass \%). The $\beta$ transus temperature is $880^{\circ} \mathrm{C}$, which is calculated using the ThermoCalc. A cylindrical sample of $12 \mathrm{~mm}$ in height and $8 \mathrm{~mm}$ in diameter were cut from the center region of the as- received hot forged disk and the thermomechanical processing shown in Fig. 1 were conducted.

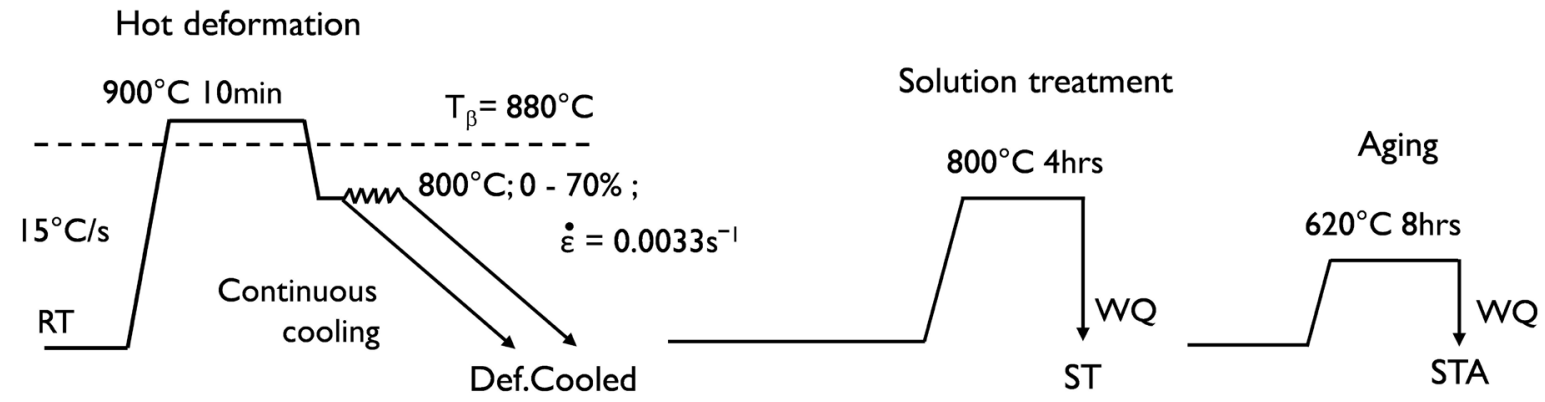

Fig.1 Thermomechanical processing of the Ti-17 alloy

The microstructures of the as-received hot forged sample were very heterogeneous. In order to, avoid the effect of such heterogeneity, the cylindrical sample was solutionized at $900^{\circ} \mathrm{C}$, slightly above the $\beta$ transus temperature for 10 min initially, to obtain homogenous $\beta$ microstructure and cooled down to $800^{\circ} \mathrm{C}$ and compressed to $0 \%, 30 \%, 50 \%$ and $70 \%$ reduction in height, at a constant initial strain rate of $0.0033 \mathrm{~s}^{-1}$ using thermomechanical simulator Thermechmastor-Z. The hot compressed samples were subsequently cooled down to room temperature at a cooling rate of $1^{\circ} \mathrm{C} / \mathrm{s}$ (Def.Cooled). Those deformed and cooled samples were 
subjected to solution treated at $800^{\circ} \mathrm{C}$ for $4 \mathrm{hr}$ followed by water quenching (ST) and then aged at $620^{\circ} \mathrm{C}$ for $8 \mathrm{hr}$ and water quenched (STA). The solution treatment and aging treatment were performed using a salt bath.

All microstructural characterizations were carried out with observation on the plane parallel to compression axis, using optical microscopy (OM), and scanning electron microscopy (SEM) with electron backscattered diffraction (EBSD) techniques. The total fraction of the $\alpha$ phase $\left(V_{\text {total }}\right)$ was quantified using X-ray diffraction from the homogenous microstructure region at the center of the thermomechanically processed samples. The volume fraction of the primary a phase $\left(V_{\text {prim- }}\right)$ by the point counting method and then the volume fraction of the secondary a $\left(V_{\text {second- } a}\right)$ was evaluated as $V_{\text {second- } a}=V_{\text {total }}-V_{\text {prim- }}$.

\section{Results and discussion}

\subsection{Microstructure of deformed and cooled specimens}

Figure 2 shows the microstructure evolution in the deformed and cooled specimens. In the $0 \%$ deformed and cooled specimen (Fig.2a), the interior of the $\beta$ grain hardly shows $\alpha$ precipitation and most of $\alpha$ are observed close to $\beta$ grain boundaries. The a phase precipitated at the $\beta$ grain boundaries is mostly in the film shape than globular (GB $\alpha$ ), whereas in the Widmanstätten plate $\alpha$ $(\mathrm{W} \alpha)$ forms close to $\mathrm{b}$ grain boundaries and also very fine in size as highlighted by the inset. Deformation at $800^{\circ} \mathrm{C}$ upto $70 \%$ does not induce recrystallization of $\beta$ except very limited fine grain formation at $\beta$ grain boundaries.
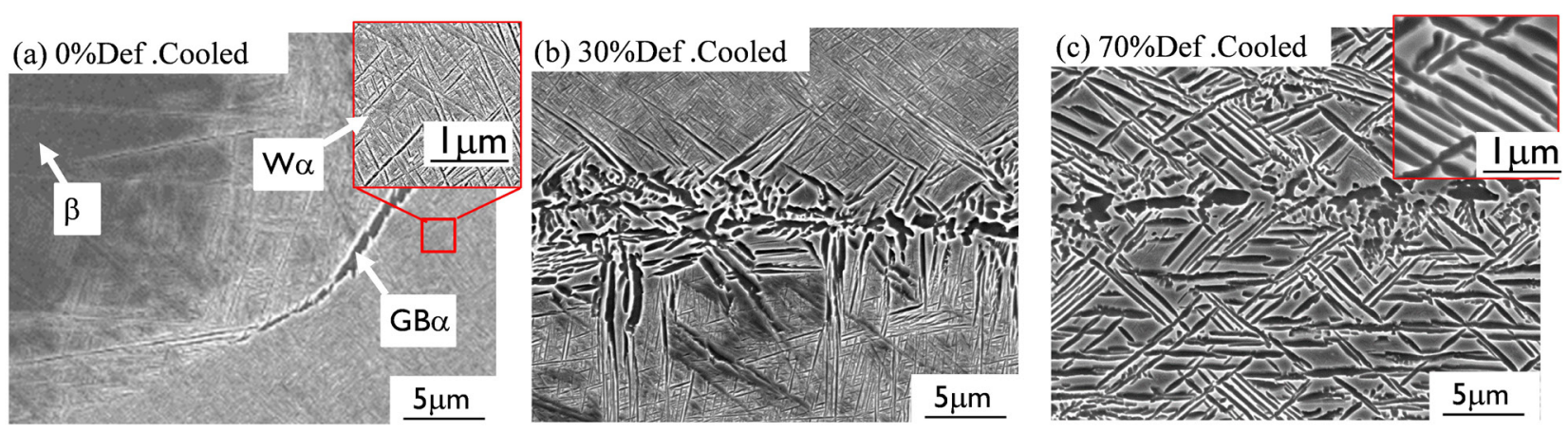

Fig.2. Microstructure of the deformed and cooled specimens (SEM).

At $30 \%$ Def. Cooled condition (Fig.2b), $\alpha$ precipitation is promoted by deformation, and most of the original $\beta$ grain is covered by the a phase. Also, $\mathrm{GB} \alpha$ becomes spheroidizied, whereas the $\mathrm{W} \alpha$ found near the $\beta$ grain boundaries are slightly coarsened compared to grain interior. At 70\% Def. Cooled condition (Fig.2c), the Wa becomes coarser than that of 30\% Def Cooled one (Fig.2c) because the higher deformation strain raised the formation temperature of $\mathrm{W} \alpha$ during subsequent cooling [10]. Therefore the difference of the $\mathrm{W} \alpha$ thickness at the prior $\mathrm{b}$ grain boundaries and within the $\beta$ grain is smaller. Subgrain boundaries introduced by deformation act as the nucleation sites for $\alpha$ precipitation resulting in the higher formation temperature of $\mathrm{W} \alpha$.

\subsection{Microstructure evolution during solution treatment and the aging process}

Figure 3a-c shows the effect of strain on the microstructure evolution during solution treatment. In the case of solution treated specimen without deformation ( $0 \%$ Def. Cooled + ST) (Fig.3a), dissolution and coarsening of $\alpha$ phase occurred in the region close to $\beta$ grain boundaries, where a phase is observed before ST; but new precipitation of $\alpha$ phase occurred in the interior of $\beta$ grain. In the deformed cooled and solution treated specimens (Def.Cooled $+\mathrm{ST}$ ), a significant amount of $\alpha$ phase is dissolved and also remained $\alpha$ is coarsened (Fig.3b and 3c). Though microstructure of the 70\% Def. Cooled specimen is coarser (Fig.1d) than 30\% Def. Cooled specimen (Fig.1b), the subsequent ST microstructure (Fig.3c) becomes quite similar in the two specimens. Figure 3d$\mathrm{f}$ shows the effect of strain the microstructure evolution during the aging process. A significant amount of finer $\alpha$ is precipitated in $\beta$ matrix surrounded by coarse $\mathrm{W} \alpha$ in all the aged specimens.. Hereafter, $\alpha$ phase exists after ST is called as primary $\alpha$ and finer $\alpha$ formed during subsequent STA is called as secondary $\alpha$. It is seen that secondary $\alpha$ is coarser at higher strain. 

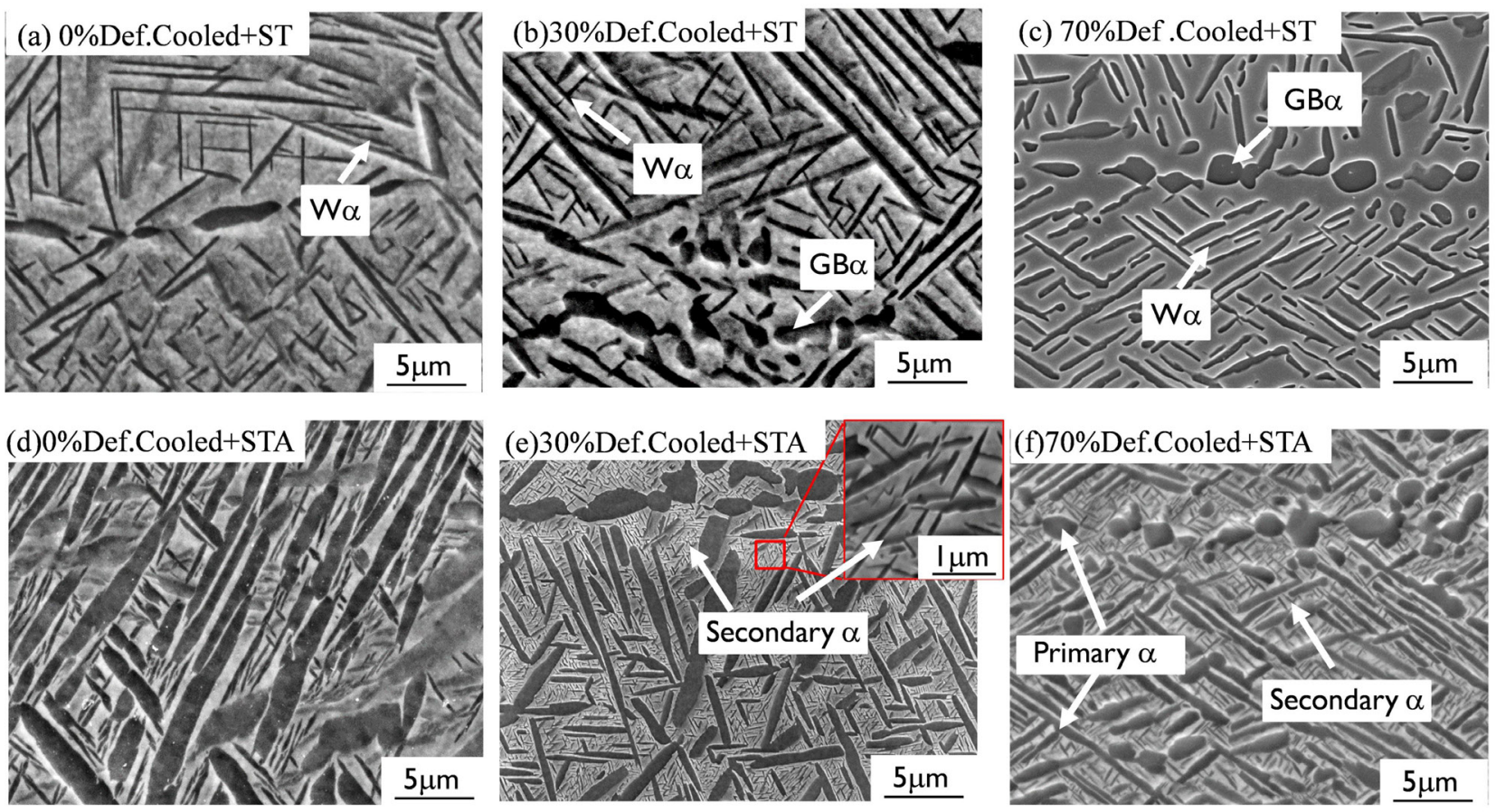

Fig.3. Microstructure evolution during solution treatment and the aging of the deformed and cooled specimens (SEM)
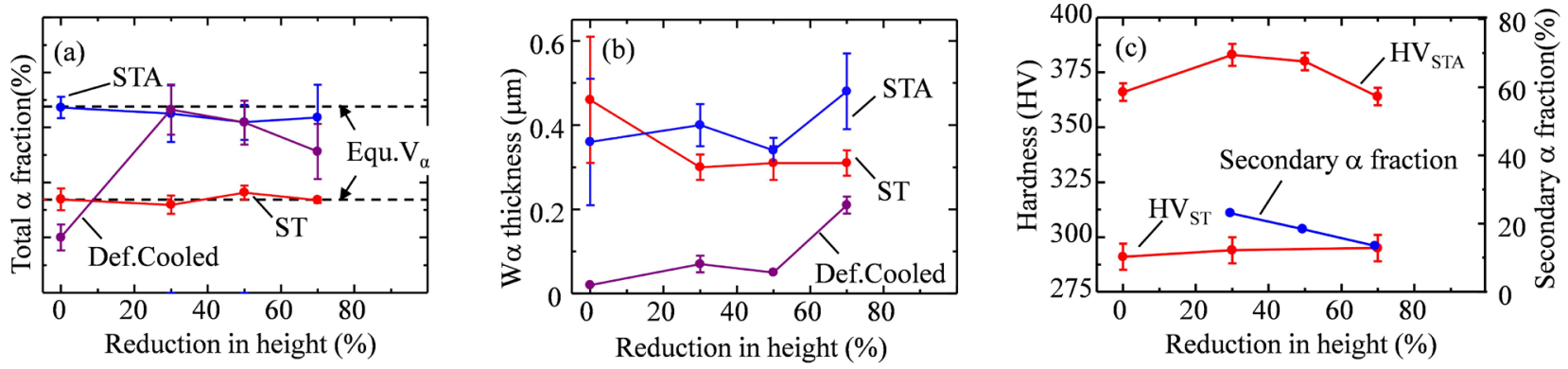

Fig.4. Effect of strain on (a) total a fraction, (b) thickness of Wa, (c) hardness and secondary a fraction during processing.

The quantified microstructure and hardness results are summarized in Fig.4. After ST, the total $\alpha$ fraction decreased in all the deformed specimens compared to Def.Cooled conditions, except in the case of UnDef.Cooled specimen where the $\alpha$ fraction found to be increased (Fig.4a). After STA, the total $\alpha$ fraction increased in all the specimens compared to ST. It is also observed that the total $\alpha$ fraction obtained after ST and STA observed nearly independent on the deformation strain, though the total $\alpha$ fraction in deformed cooled specimen depends upon the deformation strain. Furthermore, it is found that total $\alpha$ fraction after ST and STA is consistent with the equilibrium volume fraction value predicated by the empirical equation, Equ. $\mathrm{V}_{\alpha}=80.6\left\{1-\exp \left[6.834 \times 10^{-3}(\mathrm{~T}-\right.\right.$ $880)$ ]\} fitted with the observation [11]. The obtained results suggested that $4 \mathrm{hrs}$ at $800{ }^{\circ} \mathrm{C}(\mathrm{ST})$ and $8 \mathrm{hrs}$ at $620{ }^{\circ} \mathrm{C}(\mathrm{STA})$ is sufficient to reach the equilibrium fraction.

After ST, the thickness of the primary W $\alpha$ increased in all the specimen compared to Def.Cooled conditions, but the deformation strain has a lesser effect on the thickness. However, during subsequent STA, the thickness of the primary W $\alpha$ found to be increased especially at higher strain (Fig.4b). Moreover, thickening of the primary $\mathrm{W} \alpha$ during STA resulted in an increased fraction of the primary $\alpha$ and decreased secondary $\alpha$ fraction in the $70 \%$ Def. Cooled + STA compared to $30 \%$ Def.Cooled + STA (Fig. $4 \mathrm{c}$ ). It is also found that the decrease in secondary $\alpha$ fraction resulted in a decrease in measured hardness value (Fig.4c).

\subsection{Effect of deformation strain on Burgers orientation relationship (B-OR)}

Microstructure observation shown in Figs.3-4, presented that the deformation strain has a strong effect on the secondary $\alpha$ fraction through the enhanced thicknening of primary $\mathrm{W} \alpha$, is explained as follow. One possible reason is that the deformation strain affecting the elemental partitioning behavior between $\alpha$ and $\beta$ during ST. However, we confirmed that deformation strain did not show any noticeable effect on the element partitioning behavior in ST specimens. So the driving force for secondary $\alpha$ precipitation is the same in all the specimens at the beginning of the aging. Another possibility is the effect of deformation strain on the $\alpha / \beta$ interphase boundary character. It is well known that the $\alpha / \beta$ interphase satisfies Burgers orientation relationship (B-OR) which is given below [12], 


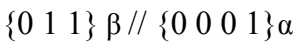

$<111>\beta / /<11-20>\alpha$

The interphase boundaries holding B-OR is highlighted as the black line in the phase map of Def.Cooled+ ST specimens deformed to a different amount of strain is shown in Fig.5.

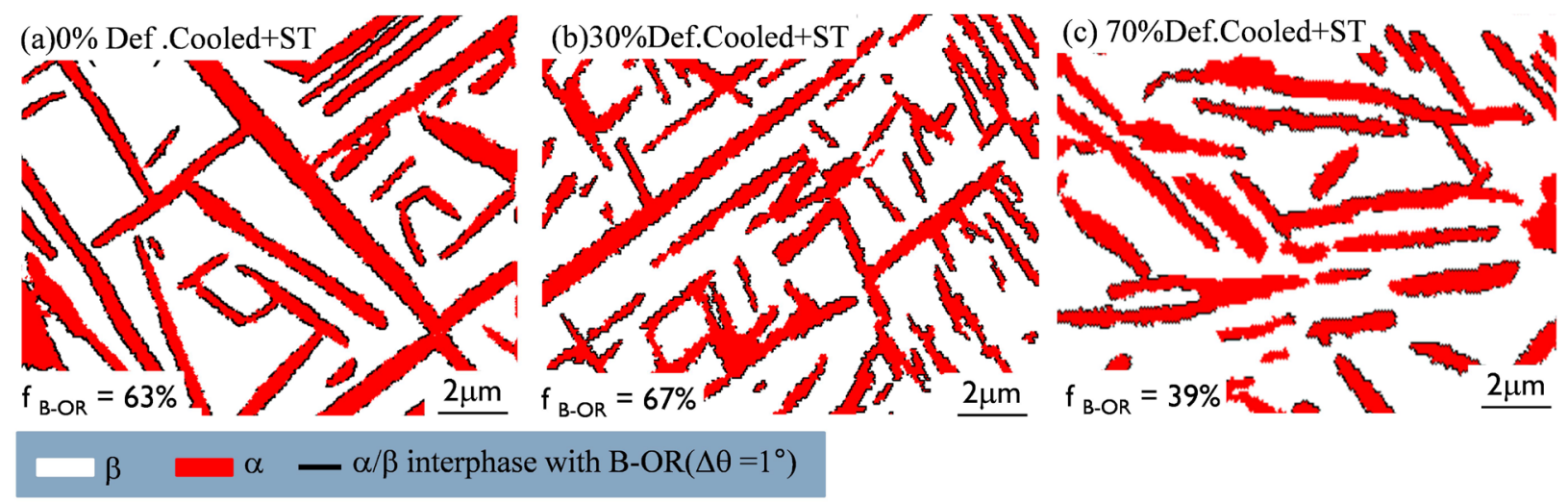

Fig.5. Effect of strain on fraction of the B-OR in Def.Cooled + ST specimens.

It can be seen that the fraction of interphase boundaries with B-OR significantly decreased by increasing the strain from $30 \%$ to $70 \%$. It indicates that the higher deformation strain decreases the fraction of B-OR fraction. Because an increase in deformation strain results in larger orientation spread in $\mathrm{b}$ matrix. Upon cooling after deformation and during subsequent solution treatment, the $\mathrm{W} \alpha$ alpha loses B-OR during its growth in the $\beta$ matrix contains high misorientations. During STA, the loss of such coherent interphase boundaries increases the mobility of the $\alpha / \beta$ boundary, which promotes the growth of primary $\alpha$. Therefore, higher interphase mobility in the deformed specimen is the primary reason for the observed increase in primary $\alpha$ fraction and corresponding decrease in secondary $\alpha$ fraction and thickening of primary $\mathrm{W} \alpha$ during aging. It is expected that the resultant change in secondary $\alpha$ fraction affect the strength after the aging treatment.

\section{Conclusion}

In a Ti-17 alloy, the microstructure evolution during solution treatment and the aging processes was studied afterdeformation below the beta transus temperature. The following conclusions are obtained,

1) Deformation strain hardly affects the total volume fraction of $\alpha$ phase during solution treatment and the aging process, which is well consistent with the equilibrium values at solution treatment and also aging temperatures.

2) Increase in the deformation strain decreases the volume fraction of secondary $\alpha$ and increases the fraction of primary $\alpha$ during aging presumably due to enhanced growth of primary $\alpha$ derived by the loss of interface coherency.

\section{Acknowledgment}

This work was supported by JST through "Cross-ministerial Strategic Innovation Promotion Program, Structural Materials for Innovation (SIP-SM ${ }^{4}$ I)".

\section{References}

[1] T. K. Redden, in R.R. Boyer and H.W. Rosenberg (eds.), Beta Titanium Alloys in the 1980s, Warrendale, PA, TMS, (1984) 239-254.

[2] R. R. Boyer, Mater. Sci. Eng. A. 213 (1996) 103-114.

[3] M. A. Greenfield, H. Margolin, Metall. Trans. 2 (1971) 841-847.

[4] T. Matsumoto, T. Nishimura, Tetsu-to-Hagane. 73 (1987) 2227-2234.

[5] H. Matsumoto, D. Naito, K. Miyoshi, K. Yamanaka, A. Chiba, Y. Yamabe-Mitarai, Sci. Technol. Adv Mater. 18 (2017) 893904.

[6] K. Yamanaka, H. Matsumoto, A. Chiba, Adv. Eng. Mater. 21 (2019) 1800775. 
[7] Z. Mu, H. Li, M. Q .Li Mater. Sci. Eng. A. 582 (2013) 108-116.

[8] J.D.C. Teixeira, B. Appolaire, E. Aeby-Gautier, S. Denis, G. Cailletaud, N. Späth, Mater. Sci. Eng. A. 448 (2007) $135-145$.

[9] J.D.C. Teixeira, B. Appolaire, E. Aeby-Gautier, S. Denis, F. Bruneseaux, Acta Mater. 54 (2006) 4261-4271.

[10] E. Chandiran, G. Miyamoto, T. Furuhara, CAMP-ISIJ 30 (2017) 1052.

[11] Y. Nagata, Master Thesis, Tohoku University (2017).

[12] W.G. Burgers, Physica. 1 (1934) 561-586.

[13] S. Y. Lee, O. Taguchi, Y. Iijima, Mater.Trans. 51 (2010) 1809-1813. 